\title{
Influenze schopenhaueriane nella Sehnsucht del giovane Horkheimer
}

\author{
Schopenhauerian influences in young Horkheimer's "Sehnsucht" \\ Patrizia Miggiano \\ Laureata in Scienze Filosofiche presso l'Università del Salento (Lecce), \\ Collaboratrice del Centro interdipartimentale di ricerca \\ su Arthur Schopenhauer e la sua scuola. \\ E-mail:patriziadmiggiano@gmail.com
}

\begin{abstract}
Riassunto: Il contributo si propone di esaminare l'influenza di Schopenhauer negli scritti del giovane Horkheimer: "Aus der Pubertät" $e$ "Dämmerung": Notizen in Deutschland, 19261931. Queste opere contengono la trattazione di una particolare attitudine interiore che caratterizza, peraltro, tutto il Romanticismo tedesco: la Sehnsucht, un anelito indefinito, tra nostalgia del passato e desiderio di felicità. Horkheimer lesse gli scritti di Schopenhauer in un periodo impregnato di questo struggente sentimento. Il pessimismo schopenhaueriano ha concesso, pertanto, a Horkheimer, di demistificare la realtà e la storia e ha rappresentato, inoltre, l'orizzonte del suo intero percorso intellettuale, influenzando la ricezione delle filosofie a lui contemporanee, in particolare il Marxismo e l'Idealismo. Comprendere l'importanza di questo orizzonte pessimistico è fondamentale per l'analisi dell'intero operato della Scuola di Francoforte.
\end{abstract}

Palavras-chave: Horkheimer; Schopenhauer; Sehnsucht; Nostalgia; Scuola di Francoforte.
Abstract: The purpose of this paper is to examine Schopenhauer's influence in young Max Horkheimer's writings: "Aus der Pubertät" and "Dämmerung": Notizen in Deutschland, 19261931. These works contain the description of a sig-nificant interior attitude that characterizes throughout the german Romanticism: the Sehnsucht, a longing between a past's nostalgia and a happiness craving. Horkheimer had read Schopenhauer's writings in this interior mood. Schopenhauer's pessimism had helped Horkheimer to demystify reality and history and therefore it had represented his whole thought's background. Furthermore this influence had shaped his reception of previous and contemporary philosophies' assumptions, in particular Marxism and Idealism. The focus on the pessimistic-schopenhauerian background is essential to find out his inspiring imprints of the whole Frankfurt School's thinking.

Keywords: Horkheimer; Schopenhauer; Sehnsucht; Nostalgia; Frankfurt School. 
«Che l'assassino non possa trionfare sulla sua vittima innocente»1.

\section{Il giovane Horkheimer e Schopenhauer: una storia da riconsiderare}

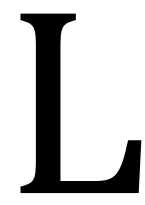

e ricostruzioni biografiche, per quanto siamo soliti constatare, si realizzano con il proposito di comporre, attraverso una serie di particolari anagrafici, di date, di luoghi, di informazioni di carattere pubblico e privato, un ritratto della vita esteriore del soggetto-oggetto della narrazione biografica. Questo consente di avvicinare il soggetto della narrazione, di conoscere più da vicino la sua parabola esistenziale e, attraverso questa conoscenza, di approfondire e decodificare alcuni aspetti della sua opera che, posti sotto questa lente, acquistano, il più delle volte, connotazioni più chiare e compiute. La visione d'insieme sul personaggio conferisce, così, un significato più o meno definito anche agli aspetti, della sua vita o della sua opera, che possono apparire, a un primo sguardo, marginali o incongruenti.

Sarebbe interessante, però, arrivati a questo punto, cercare di ricostruire la linea della vita interiore del soggetto, se ci è concesso e ne abbiamo la fortuna, attraverso le sue stesse narrazioni riguardanti desideri, sogni, riflessioni, ricordi, delusioni, malinconie, entusiasmi e tentare, attraverso queste, di intuire cosa ha mosso il personaggio nel corso della sua intera vicenda umana, quali sono stati i temi dominanti del suo cammino, su quali antiche fondamenta interiori si è edificata la sua personalità.

Questa immersione nelle profondità della vita intima del soggetto consente di puntare un faro anche sulle sue vicende esteriori - che coincidono, anzi, si potrebbe dire, rispondono ai dettami della vita interiore - poiché esso si ritrae dal profondo e la conoscenza, in questo caso, risulta diretta, senza l'intervento di alcuna mediazione. Ciò è possibile quando siamo in possesso di narrazioni, pagine e racconti scritti in prima persona, come accade nei diari o negli appunti personali e inediti, materiale di esplorazione preziosissimo.

In quest'ottica, Aus der Pubertät. Novellen und Tagebuchblätter ${ }^{2}$ e Dämmerung: Notizen in Deutschland. 1926-1931 rappresentano, per chi voglia immergersi nella vita

\footnotetext{
${ }^{1}$ HORKHEIMER, M. La nostalgia del totalmente Altro, p. 73.

2 HORKHEIMER, M. Aus der Pubertät, Novellen und Tagebuchblätter, p. 7. È lo stesso Horkheimer, nella prefazione all'opera, a raccontarci che l'insieme dei manoscritti giovanili, originariamente non destinati alla pubblicazione, venne consultato da un visitatore della sua biblioteca privata, il quale, colpito dalla raffinatezza dei contenuti e dello stile, lo invitò, nonostante le sue reticenze, alla diffusione degli scritti. 
interiore di Max Horkheimer - spiritus rector ${ }^{3}$ della Scuola di Francoforte un'occasione di conoscenza biografica irripetibile e, al tempo stesso, una testimonianza letteraria di straordinaria raffinatezza. Gli scritti giovanili, soprattutto, rivestono una particolare importanza se si vuole cogliere tutta l'eredità schopenhaueriana custodita nel pensiero del direttore dell'Istituto per la ricerca sociale, un'eredità che costituirà il Leitmotiv del suo itinerario intellettuale e, conseguentemente, anche dell'impronta che egli conferirà all'Istituto. Fazio ha messo in luce la rilevanza di una ricerca che proceda in questa direzione in "Max Horkheimer (1985-1973)»4, accogliendo l'indicazione di Schmidt sulla necessità di valutare l'influenza schopenhaueriana nell' ambito dell'intero corpus degli scritti editi - e magari anche inediti - di Horkheimer, dal momento che la stessa Teoria critica si staglia su un orizzonte fortemente schopenhaueriano.

\section{Il sentimento della separazione nella Pubertät tedesca}

Il diario giovanile di Max Horkheimer, Aus der Pubertät, desta un apprezzamento letterario e stilistico simile a quello che si risveglia alla lettura de I Buddenbrook o del Tonio Kröger di Thomas Mann - che Horkheimer conoscerà durante il suo soggiorno in California e con il quale, in seguito, avvierà un'inchiesta sui gruppi dediti alla cura dei perseguitati - o, ancora, di Demian di Hermann Hesse; ed è per questo che l'opera potrebbe, senza forzature, essere incastonata nella cornice delle novelle e dei racconti sulla formazione umana e spirituale dei giovani tedeschi di età guglielmina. L'ambiguità della dialettica interna del mondo borghese, il conflitto tra arte e vita, l'umanesimo, la salute cagionevole come origine del distacco dal mondo dei più, il rapporto con la filosofia sono i temi dominanti di queste narrazioni e ricorrono, come un timbro inconfondibile, nella vita di questi giovani figli della Germania liberal-borghese.

Un passo di grande bellezza, tratto da Tonio Kröger, così ci racconta la distanza che separa la Pubertät tedesca dai suoi padri5:

Quando comincia a diventare percepibile questa maledizione? Presto, spaventosamente presto. A un'età in cui ancora si dovrebbe vivere

\footnotetext{
3 SCHMIDT, A. «La Rivista per la ricerca sociale. Storia e significato attuale» in SCHMIDT-RUSCONI, La scuola di Francoforte, p. 11.

${ }^{4}$ Centro interdipartimentale di ricerca su Arthur Schopenhauer e la sua scuola dell'Università del Salento (a cura di), La scuola di Schopenhauer, testi e contesti, pp. 176-188.

${ }^{5}$ MANN, T. Tönio Kroger, p. 92.

Influenze schopenhaueriane nella Sehnsucht del giovane Horkheimer
} 
tranquillamente, in pace e d'accordo con Dio e col mondo. E invece lei comincia a sentirsi segnata, in un'enigmatica contrapposizione rispetto agli altri, alle persone normali, comuni, e l'abisso di ironia, di miscredenza, di opposizione, di conoscenza, di sentimenti che la separa dagli uomini si scava sempre più profondo e lei è sola e non ci sarà più possibilità di intesa. Quale destino!

Il sentimento della separazione, come Mann stesso lo definisce, è un punto di partenza straordinario per la comprensione dell'universo interiore di questa generazione tedesca.

In Demian, Hermann Hesse prende a pretesto la storia di un giovane borghese, Sinclair, per raccontare la sua personale vicenda biografica - non a caso, nei primissimi scritti, Hesse era solito firmarsi con lo pseudonimo di Emil Sinclair, usanza tipica dell'epoca, a cui anche Horkeimer, come vedremo, farà ricorso. La vita del protagonista si svolge tra molteplici snodi interiori e culturali; anch'egli portatore di istanze di ribellione nei confronti di una famiglia che interpreta l'ideale liberal-borghese mitteleuropeo in ascesa, si imbatte in un altro giovane, Demian che gli farà da guida, fino allo scoppio della prima guerra mondiale, in cui quest'ultimo perderà la vita.

L'amicizia tra i due costituisce un'isola di resistenza rispetto alle aspettative del mondo esterno, pregno di contraddizioni. L'ambiente familiare del ragazzo è improntato al rispetto delle nuove regole sociali borghesi che il ragazzo fatica a concepire come dotate di senso: la sua tensione verso un'esistenza autentica, svincolata dagli orpelli della conformazione borghese, lo tiene costantemente in uno stato di insoddisfazione e pena:

Certo, appartenevo al mondo chiaro e giusto dei miei genitori, ma dovunque volgessi l'occhio e l'orecchio, ovunque affiorava l'altra dimensione ed io vivevo anche in quella, sebbene mi fosse spesso estranea e inquietante e ne ricavassi di regola paura e la coscienza sporca. In certi periodi preferivo in assoluto vivere in quell'universo proibito e il ritorno al chiaro, per quanto necessario e buono potesse essere, spesso era quasi come rientrare in un mondo meno bello, più monotono e vuoto. A volte sentivo che scopo della mia vita era di diventare come mio padre e mia madre, come loro chiaro e puro, superiore e ordinato: ma per arrivarci il cammino era lungo, occorreva sostenere prove ed esami e la strada passava continuamente davanti all'altro mondo, quello buio ${ }^{6}$.

${ }^{6}$ HESSE, H. Demian, pp. 22-23. 
La contradditoria, ambivalente dialettica interna della classe borghese, che tanto destabilizza e confonde il giovane tedesco, poggia proprio su questo chiaroscuro descritto da Hesse: l'idea di un mondo retto, giusto, ordinato, veicolata dai dettami dell'educazione impartita dai genitori autoritari e l'agire tipico di questi stessi adulti nel proprio mondo, improntato sullo sfruttamento, la concorrenza, l'avida appropriazione. E significativamente, infatti, Hesse aggiunge: «E la cosa più strana era la contiguità di questi due mondi, e come la vicinanza li unisse! [...] L'altro mondo cominciava all'interno della nostra stessa casa»?.

Più tardi lo stesso Horkheimer, nella Prefazione di Aus der Pubertät, ricorderà la sua sensibilità di giovane ricettivo nei confronti delle incongruenze dell'ordine dominante, denunciando: «Feci esperienza del contrasto fra ciò che professavano e ciò che operavano concretamente i membri della borghesia» 8 .

La famiglia - primo messo della società — come ebbe a dire Erich Fromm molto più tardi, attua un'azione di modellamento del figlio attraverso l'educazione, forgiando i suoi desideri sulla base delle necessità del ruolo sociale atteso, finendo, così, col trasmettere «il carattere della società al neonato prima ancora che questi abbia neppure un contatto diretto con la società stessa» ${ }^{9}$.

\section{I dolori del giovane Horkheimer}

Max Horkheimer nasce il 14 febbraio 1895 a Stoccarda, figlio di un ricco industriale tessile:

La reificazione - nell'economia - a pura funzione di una grandezza economica, del patrimonio, o di un lavoro manuale o intellettuale richiesto tecnicamente, continua certo anche nella famiglia, nella misura in cui il padre si trasforma in colui che guadagna il denaro, la donna in

\footnotetext{
7 Ibidem.

8 Passo tradotto in GENINAZZI, L. Horkheimer\& Co. Gli intellettuali disorganici, p. 31. Cfr. HORKHEIMER, M. Aus der Pubertät, op. cit.in Prefazione. È utile, in proposito, non trascurare un altro passo di Horkheimer, contenuto in "Autorità e famiglia», in Teoria critica, HORKHEIMER, M. Teoria critica, p. 11: «Mentre durante il fiorire dell'epoca borghese tra famiglia e società ebbe luogo una fruttuosa interazione, per la quale l'autorità del padre era fondata sul suo ruolo nella società e la società a sua volta veniva consolidata in autorità con l'aiuto dell'educazione patriarcale, ora il ruolo della famiglia, che certamente rimane indispensabile, diventa un semplice problema di tecnica di governo. La totalità dei rapporti nell'epoca attuale, questo universale, era stato rafforzato e consolidato tramite un elemento particolare in esso universale presente, e cioè l'autorità, e questo processo si era svolto essenzialmente nella singolarità e concretezza della famiglia».

${ }_{9}^{9}$ FROMM, E. Grandezza e limiti del pensiero di Freud, p. 90.

Influenze schopenhaueriane nella Sehnsucht del giovane Horkheimer
} 
oggetto sessuale o in servo della gleba domestico, e i figli in eredi del patrimonio o in assicurazioni viventi dalle quali ci si attende la futura restituzione di ogni fatica più gli interessi ${ }^{10}$.

Questo estratto, contenuto in Studi sull'autorità e la famiglia, ben riflette le impressioni tratte dall'esperienza dell'appartenenza a un'agiata famiglia borghese, permeata da un forte orgoglio nazionale tedesco, a cavallo tra Otto e Novecento.

Certamente una visione siffatta dei rapporti interpersonali nell'ambito della famiglia ha un notevole peso per la sfera privata, il mondo interiore e l'immaginario che si andava costituendo negli anni dell'adolescenza e che troviamo raccolto nella pubblicazione Aus der Pubertät. Novellen und Tagebuchblätter.

Siamo tra il primo e il secondo decennio del Novecento, negli anni di passaggio dal proto-capitalismo al capitalismo avanzato, con il conseguente abbandono di un regime concorrenziale a favore del monopolio. Scriverà Horkheimer più tardi in proposito:

Come tutti i bambini di una famiglia della borghesia ebreo-tedesca avevo giocato docilmente ai soldatini; facevano parte del mio arsenale anche fucili con tappi di sughero e una pistola a cartucce infiammabili. Fino all'età di circa dieci anni, attorno al 1905, la domenica costituiva un giorno di gioia particolare, perché dal balcone della nostra abitazione, nella Militärstrasse, si poteva ammirare in tutta la sua ampiezza il piazzale davanti alla chiesa della guarnigione di Stoccarda. Là affluivano i distaccamenti dei vari corpi militari e affluivano un quadro animatamente colorito, fin quando nell'ordine abituale scomparivano attraverso l'ingresso della chiesa riservata a loro. [...] Erano i "nostri" soldati, appartenevano alla sfera della vita sicura, alla coscienza della pace duratura e del progresso, in cui io come gli altri bambini venivo educato ${ }^{11}$.

L'accelerata espansione capitalistica mondiale, iniziata alla fine del secolo, provocò, particolarmente in Germania, una trasformazione sostanziale del volto della classe borghese, che prima dell'impennata del capitalismo avanzato, conservava ancora una buona dose di libertà di iniziativa personale. Il padre di Horkheimer, Moritz Horkheimer, incarnava perfettamente il prototipo del nuovo borghese (il Bürgerlich) dell'imprenditore che ha consapevolezza dello slancio e della forza della posizione

${ }^{10}$ HORKHEIMER, M. «L'autoritarismo e la famiglia d'oggi» in ANSHEN, R.H. La famiglia, la sua funzione e il suo destino, p. 398.

11 La traduzione di questo passo, originariamente contenuto in KOCH, T. Porträts deutsch-jüdischer Geistesgeschichte, Poscritto di HORKHEIMER, M., è tratta da GENINAZZI, L. op. cit., p. 21. 
raggiunta ${ }^{12}$. L'ideale della realizzazione personale, attraverso la gestione della ditta familiare, dovette muoverlo anche quando sottrasse il giovane Max, allora quindicenne, dagli studi per avviarlo agli affari. Questa sarà l'occasione che offrirà al futuro direttore dell'Istituto per la ricerca sociale, la possibilità di guardare la società liberal-borghese da una prospettiva ravvicinata, prettamente economica, e che lo porterà, molti anni più tardi a scrivere:

\begin{abstract}
L'impresa stessa - che, a quanto si supponeva, sarebbe stata trasmessa in eredità ai figli dei figli - dava alle deliberazioni dell'uomo d'affari una singolare ampiezza di prospettive, in quanto egli guardava ben oltre $\mathrm{i}$ limiti della sua esistenza personale. La sua individualità era quella del produttore dei beni materiali, fiero di sé e del gruppo sociale cui apparteneva, convinto che la comunità e lo stato poggiassero su di lui e su uomini come lui, tutti dichiaratamente stimolati dall'incentivo del guadagno materiale. La sua certezza di rispondere nel modo giusto alla sfida di un mondo la cui forz animatrice era la volontà di guadagno si esprimeva nel suo ego imperioso, ma equilibrato, difensore di interessi che trascendevano le sue necessità immediate ${ }^{13}$.
\end{abstract}

Se la società liberal-borghese aveva, dunque, da un lato favorito la realizzazione economica individuale, cosa che lasciava sperare in una buona chance anche per i soggetti che fino al quel momento erano stati tagliati fuori dal processo economico, dall'altro aveva imposto un dazio da pagare: la soppressione spinta creativa individuale sotto la pressione dell' autorità razionalizzata, di cui la famiglia costituiva la cellula germinale; così «l'evoluzione del costume familiare» — rispetto al modello tradizionale - «rappresentava piuttosto la realizzazione borghese della famiglia che non la liberazione di quegli elementi di autentica emancipazione umana che nella famiglia tradizionale, nonostante la loro posizione subordinata, erano contenuti» ${ }^{14}$.

Comincia così, per il giovane Horkheimer, una profonda avversione per i meccanismi che regolavano i rapporti fra gli individui borghesi e che ne strutturavano la personalità, oltre che un vero e proprio misconoscimento della dialettica interna dell'esistenza borghese.

\title{
4. «Noi consideriamo la nostra amicizia il bene più grande. Il concetto di amicizia implica la sua durata fino alla morte ${ }^{15}$ » - Horkheimer e Pollock a Parigi
}

${ }^{12}$ Cfr. Ibidem, p. 22.

${ }^{13}$ HORKHEIMER, M. Eclisse della ragione, critica della ragione strumentale, p. 122.

${ }^{14}$ HORKHEIMER, M. Teoria critica, op. cit., p. 337.

${ }^{15}$ Questa promessa, sigillo di un'amicizia, è contenuta in un documento che risale agli anni dell'apprendisInfluenze schopenhaueriane nella Sehnsucht del giovane Horkheimer 
Era il 1912 quando Horkheimer e l'amico Pollock intrapresero, com'era usanza, un viaggio d'apprendistato lontano da casa. In occasione di questi viaggi che interessavano l'Europa, il giovane aveva l'opportunità di sperimentarsi lontano dall'ambiente familiare: era ufficialmente il primo allontanamento dal nido. La condivisione di questa esperienza con Friedrich Pollock fu particolarmente proficua. L'assidua lettura di romanzi e trattati filosofici schiuse davanti ai loro occhi un universo letterario che li colpì profondamente. Una delle poetiche più significative di quel periodo d'apprendistato fu, senza dubbio, quella del drammaturgo norvegese Henrik Ibsen, portavoce di una feroce denuncia contro gli stereotipi e le convenzioni sociali della classe borghese.

Nel 1879 Ibsen aveva, infatti, composto il dramma Casa di bambola, un quadro che ritraeva la vita coniugale di una coppia borghese in cui la donna, Nora, era ridotta a pura appendice dell'uomo. Attraverso la figura di Nora, manifesto di una condizione largamente diffusa, Ibsen invitava a una nuova consapevolezza: quella dell'essere umano che apre uno squarcio attraverso la fitta rete delle convenzioni sociali e si investe di un nuovo compito e di una nuova responsabilità, che muove dal dispiegamento ottimale di tutte le potenzialità e conduce verso la piena realizzazione. Anche August Strindberg rientra tra gli autori frequentati e apprezzati dai due giovani durante il periodo parigino.

La ricerca drammaturgica dell'autore svedese — soprattutto in Sogno del 1901 era, infatti, orientata alla riflessione sulla condizione umana: quale esistenza è possibile in un mondo che appare ostile e inospitale? La pena di esistere può essere alleviata attraverso il riconoscimento e il distacco dalle contraddizioni che abitano il mondo liberal-borghese?

[Eduard] Von Hartmann dice che l'amore è un inganno della natura, per indurre con la forza l'uomo e la donna a propagarsi. La vita mi disgusta e ha sempre fatto così. Tutto è privo di valore! Io ho osservato i miei valori e ho sofferto abbastanza. Quando ero giovane, fiducioso, idealista ero schernito. Quando divenni materialista fui perseguitato ${ }^{16}$.

tato, in cui i due giovani amici consolidarono il loro rapporto. Ne parla GENINAZZI in Horkheimer\& Co. Gli intellettuali disorganici, op. cit., p. 33.

16 Il passo è inserito nelle note drammaturgiche e registiche a Sogno di STRINDBERG, A., contenuto in Quaderni del Teatro Stabile di Torino, a cura del Centro Studi Teatro Stabile Città di Torino, p. 13. 
$\mathrm{Su}$ questa eredità si comporrà l'atteggiamento pessimistico e il successivo contraccolpo rappresentato dalla Teoria critica. Ma è con la lettura di Tolstoj che i due compagni di viaggio cementano le riflessioni sui meccanismi della vita borghese e sulle ripercussioni che questa produce: l'impossibilità di una vita naturale e il distacco dal nucleo autentico di se stessi. Questo sentimento di irreversibile perdita è descritto nel finale di La morte di Ivan Ili'ic. Il protagonista, un rispettabile magistrato gravemente ammalato, si trova a riflettere sulla sua condizione, a poche ore dalla sua morte:

Gli venne in mente che i suoi timidissimi tentativi di ribellione contro ciò che la gente dell'alta società considerava buono, tentativi appena abbozzati, ch'egli s'era sempre affrettato a reprimere, potevano essere quelli autentici, e tutto il resto, errore. [...] Quando al mattino rivide il domestico, la moglie, la figlia, il dottore, si accorse che ogni loro movimento, ogni loro parola confermavano la terribile verità che aveva scoperto durante la notte. In loro vedeva se stesso, tutto ciò di cui aveva vissuto, e vide con chiarezza che era sbagliato, un enorme, terribile inganno che nascondeva la vita e la morte. Questa consapevolezza aumentava, decuplicava le sofferenze fisiche ${ }^{17}$.

Lo sguardo sulle contraddizioni dell'ordine dominante, formatosi in quegli anni di frenetiche letture condivise con l'amico, si congiunse presto all'intuizione di una possibilità alternativa all'esistente: La critica della ragion pura forgiò il sogno di una felicità possibile grazie al ricorso alla critica come strumento di conoscenza autentica e libera da condizionamenti. Portare l'esistente dinanzi al tribunale della ragione poteva significare ridiscuterlo, sminuzzarlo e osservarne le aporie. Questo era il primo passo per una possibile, futura felicità del genere umano. Il rifiuto di un'accettazione immediata e passiva della realtà, ma soprattutto l'atto della scomposizione e della riflessione su di essa, costituisce una premessa fondamentale per i successivi snodi dell'opera horkeimeriana e dell'intero Istituto per la ricerca sociale.

\section{Pollock presenta Schopenhauer ad Horkheimer}

Sempre durante il viaggio d'apprendistato Pollock consigliò al giovane Horkheimer la lettura di un particolare libretto, opera di un filosofo che segnerà come nessun altro la vita e l'opera del futuro direttore dell'Istituto. Si trattava di Aforismi sulla saggezza di vita di Arthur Schopenhauer. 
Il pessimismo metafisico [...] mi è sempre stato familiare. Il mio primo contatto con la filosofia lo devo all'opera di Schopenhauer; il rapporto con la dottrina di Hegel e Marx, la volontà di comprendere e di modificare la realtà sociale, non hanno - nonostante il contrasto politico - cancellato l'esperienza che ho tratto dalla sua filosofia ${ }^{18}$.

Il ritratto della condizione umana custodito nelle pagine degli Aforismi scosse profondamente il giovane Horkheimer. L'arguzia con cui, in quell'opera, veniva rappresentata la lenta scoperta dell'infelicità come dimensione intima e caratterizzante di ogni esperienza umana, offrì ad Horkheimer il terreno su cui edificare la sua personalità di giovane intellettuale.

Ecco pure un sentimento che contribuisce alla felicità dell'infanzia: come nei primi giorni di primavera qualunque fogliame ha lo stesso colore e quasi la stessa forma, così nella prima giovinezza ci rassomigliamo tutti, e andiamo d'accordo perfettamente. Non è che colla pubertà che comincia la divergenza, la quale va sempre aumentando, pari a quella dei raggi d'un cerchio. Ciò che turba, ciò che rende infelici gli anni di giovinezza, il rimanente di questa prima metà della vita tanto preferibile alla seconda, si è la caccia alla felicità intrapresa nel fermo convincimento che la si possa trovare nell'esistenza. Ecco la fonte della speranza sempre delusa, che genera a sua volta lo scontento. Le immagini ingannatrici d'un vago sogno di felicità volano davanti gli occhi nostri sotto forme capricciosamente scelte, e noi cerchiamo invano il loro tipo originale. Perciò siamo durante la giovinezza quasi sempre mal soddisfatti del nostro stato e del nostro ambiente qualunque si siano, perocchè ad essi attribuiamo ciò che dovremmo sempre riferire alla inanità od alla miseria della vita umana, colle quali allora facciamo conoscenza per la prima volta, dopo esserci aspettati ben altra cosa ${ }^{19}$.

L'influenza che Schopenhauer esercitò su Horkheimer, a partire da questa lettura e per tutto il corso della sua vita, è tema abbastanza complesso dal momento che non si tratta di un'influenza esclusivamente teorica e metodologica, bensì di un vero e proprio orizzonte, pessimistico e demistificatore, che, come puntualizza Geninazzi, funge da

punto coagulante di una serie di sentimenti caratteristici dell'ambiente culturale, e in questo orizzonte Schopenhauer va posto accanto a Kierkegaard e Nietzsche, come un antenato degli indirizzi della "filosofia della vita" e più tardi dell'esistenzialismo, anzi come uno degli autori in

18 HORKHEIMER, M. Teoria critica, op. cit., p. 11.

${ }^{19}$ SCHOPENHAUER, A. Aforismi sulla saggezza del vivere, pp. 105-106.

Influenze schopenhaueriane nella Sehnsucht del giovane Horkheimer 
cui i concetti di angoscia e di scacco esistenziale non vengono usati ideologicamente ${ }^{20}$.

Lo Jammer des Lebens con Schopenhauer si accosta, dunque, ad una visione pessimistica, non più solo dell'esistenza umana, ma dell'intero corso del mondo e della storia, così come pure dell'essenza del cosmo.

Negli scritti giovanili - lettere, novelle e pagine di diario, nelle quali la protesta morale nei confronti dell'ingiustizia sociale si unisce ad una visione pessimistica del mondo - la presenza di Schopenhauer è centrale. Essi anzi «contengono in modo abbastanza sorprendente, i lineamenti di una concezione metafisica che elabora e in alcuni casi prosegue la dottrina di Schopenhauer» ${ }^{21}$.

Questo primo accostamento al pensiero schopenhaueriano fece sì che, in Horkheimer, quel dissidio con la società del suo tempo - che l'esperienza familiare aveva già in precedenza ispirato - prendesse la forma di una vera e propria coscienza alternativa all'esistente, di un'ansia di vivere.

L'aggravarsi delle tensioni internazionali e il conseguente scoppio della prima guerra mondiale non fecero che esasperare, nel giovane, i sentimenti di protesta e di odio verso quell'evento dalle dinamiche inaccettabili, che aveva creato in lui vuoto e smarrimento:

Allora arrivò la prima guerra mondiale, e io l'ho odiata fin dal primo giorno. Non perché avessi aderito ad una teoria pacifista o comunque politica, ma perché, nonostante l'indignazione per l'assassinio di Sarajevo, spiegazioni, come quelle delle bombe a Norimberga, non suonavano convincenti [...] Avevo visto Parigi e Londra e non potevo credere che in questi posti gli uomini fossero tanto più amanti della guerra che non il nostro imperatore amante della pace e fossero tanto più cattivi di me che avrei dovuto mettermi a sparare su di loro [...] La mia fede negli insegnamenti nella casa paterna sul Reich tedesco cominciò a vacillare ed ebbi la sensazione che qualcosa di orribile, qualcosa a cui non si sarebbe mai potuto rimediare stesse accadendo all'Europa, all'umanità addirittura. La cosa più terribile mi sembrò senza che allora per altro fossi riuscito a formularla - che il compito storico, per così dire, la missione dei popoli europei, ed in particolare di

${ }^{20}$ GENINAZZI, Horkheimer \& Co. Gli intellettuali disorganici, op. cit., p. 34.

${ }^{21}$ Centro interdipartimentale di ricerca su Arthur Schopenhauer e la sua scuola dell'Università del Salento (a cura di), La scuola di Schopenhauer, testi e contesti, op. cit., p. 178. Il passo tra virgolette è la traduzione di un estratto tratto da SCHMIDT, A. Frühe Dokumente der Kritischen Theorie, in HORKHEIMER, M. Aus der Pubertät. Novellen und Tagebuchblätter, op. cit., p. 367: «Zugleich aber enthalten sie, erstaunlich genug, Umrisse einer metaphysichen Konzeption, die Schopenhauers Lehre verarbeitet und - teilwiese fortbildet».

Influenze schopenhaueriane nella Sehnsucht del giovane Horkheimer 
quello tedesco, cui appartenevo, fosse irrimediabilmente sacrificato ${ }^{22}$.

Horkheimer fu chiamato alle armi nel 1917, ma, a causa del suo stato di salute, non partecipò alle operazioni belliche. Venne ricoverato in una clinica, da dove, grazie alle notizie degli amici, seguiva l'andamento del conflitto.

Nel frattempo il dissidio con il padre era destinato a radicalizzarsi perché, proprio in quel periodo, il giovane comunicò la sua decisione di voler sposare Rose Riekher, la sua Maidon, segretaria della ditta di famiglia, proveniente da una famiglia di fede cristiana.

Anche in questo caso i motivi biografici e familiari si intersecano strettamente con le vicende che premono sulla situazione sociale e politica del tempo e si pongono all'origine di un profondo conflitto nell'animo del giovane al bivio tra un'esistenza autentica - che tenesse conto dell'amore per la filosofia, per la verità e per Maidon - o l'asservimento alla volontà del padre e del sistema borghese che lo voleva efficiente e produttivo nella fabbrica di famiglia. A questi giorni tormentati risalgono i pensieri:

Questa guerra e tutte le sofferenze sono necessarie. Impennarsi contro la necessità è pazzia. Si deve imparare a sopportarla. Sì, questo è lo scopo della nostra esistenza. Devo rinunciare alla pretesa di felicità personale; non posso più cercare di emergere su ciò che è comune: devo soccombere per esso e in esso. Finora io volevo egoisticamente il bene della mia propria persona, del tutto insignificante. [...] Tutto è buono, tutto è buono, solo io sono cattivo. Io non riuscirò a liberarmi da questo sentimento opprimente 23 .

E ancora, in un bellissimo passo, sempre da Aus der Pubertät:

Ieri mi trovavo nella nostra filiale di Hirsau. In mezzo ai boschi, presso un ruscello, hanno costruito alcune case con complicate macchine all'interno. Nel calore torrido stanno alcuni poveri uomini e lavorano col volto sudato e arrossato. Devo prendere parte al lavoro di queste persone e di mio padre e perciò devo mettere da parte le mie necessità vitali, per produrre denaro e cotone artificiale o qualcosa di simile - no, no, no, è impossibile: ciò significherebbe la morte della mia anima nostalgica, il consumarsi del mio cuore assetato. Voglio fare ciò che la

22 La traduzione è tratta da PONSETTO, A. Max Horkheimer, dalla distruzione del mito al mito della distruzione, p. 31, ma il passo è originariamente contenuto in KOCH, T. Porträts deutsch-jüdischer Geistesgeschichte,, op.cit. pp. 256-265.

${ }^{23}$ HORKHEIMER, M. Aus der Pubertät, op. cit., p. 155: «Alles ist gut, alles ist gut - nur ich bin schlecht: diese Worte wurde ich beim Erwachen nicht los, sie verfolgten mich während des Ankleidens und auf meinem Wege durch die feuchten nebeligen Straßen».

Influenze schopenhaueriane nella Sehnsucht del giovane Horkheimer 
mia volontà, che ho chiara, mi ingiunge. Voglio vivere e ricercare, assecondando la mia tensione verso la verità, conoscere ciò che desidero, aiutare l'oppresso, saziare il mio odio verso l'ingiustizia e sconfiggere i farisei, ma soprattutto cercare l'amore, cercare amore e comprensione, di cui ogni fibra di me stesso è assetata 24 .

L'incertezza tra rifiuto e accettazione incondizionata si riflette molto bene in una raccolta di lettere, intitolata significativamente Krieg, in cui Horkheimer immagina la corrispondenza tra una coppia di fidanzati, durante il periodo della guerra: lui è al fronte e descrive a lei gli orrori che si presentano davanti ai suoi occhi; lei lo conforta e si conforta con l'idea della necessità di questi eventi, inscritti nel naturale corso delle cose, contro cui il singolo nulla può. Solo alla notizia della morte del giovane, la ragazza maturerà un sentimento di profonda avversione per la guerra. Anche nei due personaggi di Krieg, dunque, i motivi personali si intersecano e modellano le opinioni sulle vicende umane e, in generale, sulla storia.

Questa filosofia della crisi, che smuove profondamente il giovane Horkheimer e il sistema di convinzioni e valori ereditato dalla famiglia e conservato fino a quel momento, emerge ancora in un altro estratto del suo Nachla $\beta^{25}$ :

La verità, che parla a partire dal mondo in modo chiaro e inequivocabile, ha sopraffatto, di colpo, anche i dogmi ebraici impressi in me, col risultato, per me, che questo mondo non poteva essere opera di un'essenza universalmente buona, ma, piuttosto, opera di un demone che ha chiamato all'esistenza le creature per deliziarsi alla vista delle loro pene; sono i dati concreti che fanno pensare a questo, perciò mi assalì la convinzione che le cose stanno così in questo modo.

Come già accadde per Schopenhauer, anche Horkheimer, davanti alla scoperta dell'ineludibilità del dolore nel mondo, interiorizza la convinzione, suffragata, tra l'altro, dagli orrori della guerra, dell'insensatezza del reale e la rivendica proprio nella denuncia di quegli ideali borghesi - libertà e ancor più sicurezza di vita - che sono andati perduti nel conflitto bellico e in nome dei quali il pensiero borghese aveva legittimato l'ingiustizia e la disuguaglianza, presentandoli come mezzi necessari per il conseguimento del successo personale di pochi, a scapito della felicità di molti.

L'aspirazione ad una verità autentica, libera dai condizionamenti del suo tempo,

\footnotetext{
${ }^{24}$ Originariamente in HORKHEIMER, M. Aus der Pubertät, op. cit., è tradotto nel testo di PONSETTO, A. Max Horkheimer, dalla distruzione del mito al mito della distruzione, op. cit. p. 33.

25 Ibidem.

Influenze schopenhaueriane nella Sehnsucht del giovane Horkheimer
} 
però, ancora si contrappone al tormento derivante dalla tentazione di cedere dinanzi alle istanze del mondo borghese - incarnate dall'ambiente familiare - e rinunciando a se stessi e alla propria natura segnata da un anelito indefinito: la Sehnsucht, sentimento cardine dell'esperienza giovanile e dell'intero spirito romantico tedesco.

Sehnsucht (da Sehnen «desiderare» e Sucht «brama, dipendenza») è il sentimento dello struggimento, dell'anelito verso un approdo indefinito - e forse irraggiungibile dell'umano vagare. È, dunque, una dimensione senza tempo poiché si pone sul doppio binario della nostalgia di un passato paradiso perduto e del desiderio straziante di felicità futura. Il tempo, nel suo doppio vettore di passato e futuro, diventa, così, custode di una speranza di realizzazione e accoglimento che al contempo, sancisce una condanna autoinflitta: l'impossibilità di radicarsi felicemente nel presente. In Aus der Pubertät è sempre presente e viva quest'esperienza interiore, questo sentimento di insoddisfazione che si radica nella coscienza infelice del giovane Horkheimer e prende la forma della tensione e del sentimento della mancanza, che tenta di trovare rifugio nell'ideale della promesse de bonheur:

Der heutige Tag ist das Symbol meines gegenwärtigen Lebens. Ich habe die glänzendste Stellung mit einer noch glänzenderen Zukunft im Geschäft meines Vaters, kann mir jedes Vergnügen erlauben, das mich reizt, kann mich in die geschäftliche Arbeit vertiefen, kann mich zerstreuen oder meinen Liebhabereien nachhängen - und doch verzehrt mich die Flamme der brennendstan Sehnsucht. Unkluge, unvernünftige Gedanken ziehen mich hinaus in eine Welt, wo ich die Sonne wähne, die doch droben am blauen Himmel hängt, die wir nicht anschauen können, ohne geblendet zu werden ${ }^{26}$.

Il termine Sehnsucht, come vedremo, acquisirà con Horkheimer, un senso molto complesso. Egli riprenderà questo termine anche nel titolo del suo ultimo scritto, Die Sehnsucht nach dem ganz Anderen, quando ripenserà la forma dei rapporti tra gli individui e la storia. Questo ci conduce a pensare che la Senhsucht sia il panorama di fondo dell'intera opera horkheimeriana e che la critica e la discussione dell'ordine dominante si siano incastonate, in seguito - soprattutto negli anni della Teoria critica proprio in questa cornice. In questi primi scritti giovanili, il termine viene impiegato, però, con una sfumatura leggermente differente: si potrebbe dire si tratti di una Sehnsucht proiettata al futuro come desiderio di felicità ed emancipazione e che, dunque, 
lo sfondo emotivo che caratterizza il primo Horkheimer non è la nostalgia in senso letterale, bensì la melanconia struggente (Jammer des Lebens in Schopenhauer)27:

[...] du allein willst Mensch sein und nicht Mörder? Leben und nicht töten? Und meinst, Gott segne deine Sehnsucht? Wei $\beta$ t du nicht, da $\beta$ Gott dies Schauspiel liebt; da $\beta$ er nur den zu sich nimmt und heiligt, der nicht mehr widerspricht, sich fügt und begnügt und ja sagt; da $\beta$ jeder Mensch verflucht ist?28

Geninazzi osserva che:

in alcuni casi, soprattutto in alcune novelle e racconti, è visibile il gusto tardoromantico, in Germania ancora molto vivo per l'influsso dell'opere wagneriane, per i sentimenti oscuri e misteriosi che fanno prorompere nei protagonisti passioni impetuose ${ }^{29}$.

Il racconto di un'epoca e di una generazione attraverso questa inclinazione all'intimo struggimento, consente di comprendere a pieno gli snodi successivi dell'opera horkeimeriana. Questa sofferenza, infatti, condurrà a una riflessione sull'esistente, sull'umano e sulla storia. Il dolore, nella forma del rifiuto dell'ordine dominante, comporta inevitabilmente l'impossibilità di un inserimento acritico nel mondo. Dalla crisi alla critica, dunque, dal patire all'agire, attraverso un'evoluzione della propria postura nel mondo.

\section{L'indelebile impronta schopenhaueriana}

Lo sfondo del pessimismo schopenhaueriano, in Horkheimer, integra la personale contrapposizione tra spirito e materia, consentendo di scongiurare il rischio dell'ipostatizzazione e di una «teorizzazione compiuta della disperazione e della morte, come tragicità dell'esistenza. Egli già dai primi anni ha saputo cogliere e mantenere quella sobrietà schopenhaueriana di fronte al nulla, senza aggettivi» ${ }^{30}$. L'orizzonte pessimistico, però, non raggiunge mai il livello di un rifiuto espresso, ma appare, piuttosto, come insofferenza, come «tensione non risolta tra ideale e reale» ${ }^{31}$. Questo

\footnotetext{
27 SCHOPENHAUER, A. SW, p. 39.

${ }^{28}$ HORKHEIMER, M. Aus der pubertät, op. cit., p. 33.

${ }^{29}$ GENINAZZI, L. Horkheimer\& Co. Gli intellettuali disorganici, op. cit, p. 38.

30 GENINAZZI, L. Horkheimer\& Co. Gli intellettuali disorganici, op. cit., p. 49.

${ }^{31}$ Idem, p. 42.

Influenze schopenhaueriane nella Sehnsucht del giovane Horkheimer
} 
perché, nonostante lo scontro con gli avvenimenti tragici del suo tempo e la conseguente rielaborazione in chiave pessimistica, rimane pur sempre vivo il desiderio di discussione dell'ordine esistente, che porta Horkheimer a non accettare come plausibile una qualsivoglia giustificazione del reale che, in qualche modo, possa legittimare il sopruso e l'ingiustizia. Così si esprime Horkheimer in quel periodo ${ }^{32}$ :

Das Positive, das Bestehende ist immer schlecht, doch seine Beschaffenheit ist der einzige Anhaltspunkt, den unsere Sinne benützen können, um das Geistige aus ihm herauszufühlen, das wir nicht greifen können und das seine mehr oder minder große Schönheit ausmacht. Bestände die Körperwelt nicht, so wäre das $M a \beta$ der Schönhiet voll, doch für uns gäbe es nur ein großes Nichts - Nirwana.

Occorre, però, soffermarci su questo punto, poiché sono necessarie delle precisazioni: se con Schopenhauer si parla di metafisica pessimista — che permea tutto il reale nella totalità dei suoi aspetti e non propone prospettive di soluzione - per Horkheimer il discorso si compone in maniera differente: il suo pessimismo è ambivalente dal momento che in alcuni passi dell'opera acquista toni profondamente malinconici, quasi disperati (come nei passi precedentemente riportati); in altri è il fondamento di uno slancio di speranza e desiderio di ricomposizione dell'ordine esistente, molto lontano dal rifiuto schopenhaueriano. In altre parole, l'animo del giovane Horkheimer, in alcune pagine, racconta di un profondo tormento, di una cocente delusione, di un abbandono da parte di un Dio, se così vogliamo dire, che non è essenza buona, ma demone, natura maligna che gioisce delle disavventure del genere umano. Altrettanto presenti e significativi, sono, però, i momenti in cui questo senso di abbandono si converte in protesta e volontà di impegno. Il pessimismo di Horkheimer, dunque, è un pessimismo che rifiuta la rassegnazione. Illuminante, per comprendere l'orizzonte teorico pessimistico del futuro direttore dell'Istituto per la ricerca sociale, sarà una sua dichiarazione, contenuta in Teoria critica Ieri e oggi: «Era questo il nostro principio: pessimisti in teoria e ottimisti nella pratica» ${ }^{33}$. La pratica si traduce in uno slancio attivo di ridiscussione e cambiamento dell'ordine dominante, nell'ideale vivo, sempre presente, della fratellanza e dell'amore tra gli uomini, nel livellamento delle ingiustizie sociali, nel rifiuto della mercificazione del lavoro e di ogni attività umana,

32 HORKHEIMER, M. Aus der Pubertät, op.cit, p. 22.

33 HORKHEIMER, M. Teoria critica Ieri e oggi, in La società in transizione, p. 180.

Influenze schopenhaueriane nella Sehnsucht del giovane Horkheimer 
nell'ostinata ricerca e riaffermazione della verità anche in condizioni storico-politiche che sembrano averla irreversibilmente compromessa, nella pietas che deve ricondurre la storia degli uomini sulla strada del miglioramento delle proprie condizioni. Questa stessa pietas costringe Horkheimer ad aprire gli occhi sulle terribili condizioni degli operai tedeschi di quegli anni, compresi quelli che prestavano lavoro nella ditta del padre. Scrive in una lettera datata luglio 1916 al cugino Hans, a proposito di Katharina Krämer, sua dipendente:

\begin{abstract}
Destatasi alla vita da una famiglia di nove persone, figlia non desiderata, decima a soffrire la fame, con lo stomaco che continuamente brontola o che selvaggiamente grida; costretta in un puzzolente appartamento di due stanze a giocare a giochi senza senso sui tre scalini sotto la porta di casa; trattataogni giorno come stupida e fannullona, è messa alla berlina di fronte a 82 compagni di scuola; venduta a causa di una gravidanza disonorevole a un portatore di letame ubriaco, che con allegri squilli di tromba è costretto ad andare a lavorare sul fronte russo e che viene spedito in Siberia, dopo essere stata liquidata dall'ufficio di assistenza con l'ironia di un sussidio quotidiano di un marco per tre persone; inviata da una parte all'altra dei vari uffici, finalmente dopo essersi nutrita per 14 giorni di patate, trova lavoro in un ambiente orrendo, dall'aria densa e insopportabile, stantia di polvere e di mutande puzzolenti; felice di poter comprare per il giorno seguente la paga del latte a sufficienza per i bambini, che coi morsi della fame tendono le braccia verso la madre; prostrata e abbruttita dall'epilessia e condannata senza fine a vivere di patate ${ }^{34}$.
\end{abstract}

La solidarietà per gli oppressi - le cui istanze di emancipazione Horkheimer sente vicine ed urgenti - è significativa non solo perché esprime sentimenti molto densi di umanità e compassione, ma anche perché sarà la soglia della sua particolare adesione al marxismo. Particolare perché non può mai essere affrancata dallo sfondo pessimistico-schopenhaueriano. Ecco perché si può dire che la lente di ingrandimento attraverso cui Horkheimer guarda, legge e interpreta i fatti del Novecento, come anche il modo in cui egli recepisce le filosofie della storia a lui precedenti e contemporanee idealismo e materialismo fra tutte - risentano dell'impronta demistificatrice schopenhaueriana, presente, come abbiamo visto, fin dalle letture dell'adolescenza. Proprio quest'impronta costituisce un nucleo costante, che rimane invariato nonostante i molteplici snodi intellettuali e biografici del filosofo.

L'individuazione di questo filo conduttore di matrice pessimistico-

34 Passo originale contenuto in GUMNIOR H. e RINGGUTH, R. Max Horkheimer, p. 11, poi tradotto in GENINAZZI, L. Horkheimer\& Co. Gli intellettuali disorganici, op. cit., p. 49. 
schopenhaueriana è un'opera di particolare rilevanza, se si pensa che rintracciare i motivi ispiratori del pensiero di Horkheimer, significa necessariamente entrare in possesso di una chiave di lettura più completa per l'intero operato della Scuola di Francoforte, dal momento che, come riteneva lo stesso Alfred Schmidt, suo discepolo, «la storia dell'Istituto di Francoforte coincide in larga parte con la biografia intellettuale di Horkheimer ${ }^{35}$ ». L'indelebile traccia schopenhaueriana si pone, dunque, di volta in volta come prima e indiscutibile premessa per le successive evoluzioni del pensiero horkheimeriano e risulta fortemente evidente anche nei suoi ultimi anni di attività, come si evince dalle pagine dei suoi ultimi scritti, Taccuini 1950-1969 e La Nostalgia del totalmente Altro.

È interessante, per delineare la vicinanza con Schopenhauer, evidenziare anche l'affinità biografica tra i due autori. Come aveva già notato Schmidt nella Postfazione di Aus der Pubertät - di cui curò la pubblicazione l'anno seguente la morte di Horkheimer - sono, infatti, molteplici i tratti che li accomunano: entrambi figli di industriali che speravano di indirizzarli al lavoro nella ditta di famiglia, si avvicinano alla filosofia, pur avendo manifestato delle attitudini organizzative per la gestione economica della fabbrica.

La vocazione alla ricerca della verità, però, ha la voce di un sirena ed è impossibile resisterle. Scriverà Horkeheimer in quei giorni, accorciando sempre più la distanza da Schopenhauer ${ }^{36}$ :

Ho davanti agli occhi quel grande, magnifico, inconcepibile Nulla, come l'orizzonte il cui vuoto appare come un blu bellissimo, come la quintessenza di ogni felicità, come la meta dei nostri desideri; se noi potessimo volargli incontro, il blu diventerebbe sempre più scuro, fino $\mathrm{a}$ traformarsi in nero, in nulla.

Più tardi, in un saggio pubblicato nel 1961, dal titolo «L'attualità di Schopenhauer ${ }^{37}$, Horkheimer riassumerà, con parole significative, questa visione del

\footnotetext{
${ }^{35}$ SCHMIDT, A. «La Rivista per la ricerca sociale. Storia e significato attuale» in SCHMIDT-RUSCONI, La scuola di Francoforte, op. cit. p. 12.

36 HORKHEIMER, M. Aus der Pubertät, op. cit., p. 11, tradotto in L. GENINAZZI, Horkheimer\& Co. Gli intellettuali disorganici, op. cit., p. 50.

37 «Die Aktualität Schopenhauer» (in «Schopenhauer Jahrbuch für das Jahr 1961», pp.12-25; ristampato in Sociologica II, cit., pp. 124-141. La traduzione italiana della «Attualità di Schopenhauer» si trova nell'antologia La scuola di Schopenhauer, testi e contesti, a cura del Centro interdipartimentale di ricerca su Arthur Schopenhauer e la sua scuola dell'Università del Salento, op. cit., pp. 526-545. In HORKHEIMER, M. 
mondo che a tratti si costituì, nei suoi scritti giovanili, come una vera e propria metafisica, come processo conoscitivo del reale: «Per Schopenhauer la giustizia della posterità era garantita da quella storia spesso detestata. Questa giustizia è la sua nostalgia, la sua utopia» ${ }^{38}$...e anche quella di Horkheimer.

\title{
7. Dämmerung: Notizen in Deutschland - 1926-1931
}

Come abbiamo visto, l'avvicinamento alla fabbrica del padre è occasione d'incontro tra la critica all'ordine capitalista e l'urgenza del problema del proletariato, cui Horkheimer arriva attraverso l'esperienza diretta della Germania rivoluzionaria, della prima guerra mondiale e della rivolta proletaria nel novembre 1918 a Monaco. Scrive Alfred Schmidt in riferimento a quegli anni:

\begin{abstract}
Durante la libera docenza, dal 1926 al 1930, Horkheimer riesce a liberarsi dai condizionamenti accademici e a sviluppare quel concetto di filosofia che gli sarà in seguito caratteristico. Questa [...] fase del suo pensiero è contrassegnata dalla decisa adesione al marxismo, la cui forza esplosiva gli si era probabilmente fatta già evidente durante gli avvenimenti rivoluzionari del primo dopoguerra. Il radicalismo maturato dalla coscienza politica di Horkheimer si riflette nel volume di aforismi Dämmerung [Crepuscolo], pubblicato in Svizzera solo nel 1934 con lo pseudonimo di Heinrich Regius ${ }^{39}$.
\end{abstract}

Ma qual è l'itinerario personale che conduce Horkheimer all'adesione al marxismo e con quali caratteristiche?

La permanenza nella fabbrica del padre fu fonte di riflessione sul ruolo che il proletariato stava guadagnandosi e condusse Horkheimer alla conclusione che ogni regime fondato sul dominio e sulla diseguaglianza porta in seno la sua condanna, che si manifesta in forma di rivolta dirompente una volta che le masse acquistano coscienza di questa condizione. Sono queste le premesse per l'adesione al marxismo, già illustrate in un racconto contenuto ancora in Aus der Pubertät, dal titolo Jochai:

Allorché gli inferni delle fabbriche vomitarono i loro dannati in foggia di demoni sui sazi borghesi; allorché, per la prima volta, la città vide con

Zur Kritik der instrumentellen Vernunft, ed. da SCHMIDT, A. pp.248-268; ora in HORKHEIMER, M. Vorträge und Aufzeichnungen 1949-1973, in Gesammelte Schriften, 10 Bde., pp. 122-142.

38 Ibidem, p. 526.

39 M. HORKHEIMER, Taccuini 1950-1969, op. cit., p. 19.

Influenze schopenhaueriane nella Sehnsucht del giovane Horkheimer 
occhi terrificati la ribellione (sulla piazza del mercato il lastricato rosso, l'aria densa del fragore e dell'incendio infernale, la periferia paurosamente silenziosa, ogni villa con le luci spente e il fiato sospeso), due uomini stavano nella semioscurità - appena visibili l'uno all'altro, separati dalle opere di fortificazione dei loro demoni e dalla cecità derivante da un'educazione che attizza l'odio. Jochai, il "Commis" non aveva potuto sparare, era fuggito di là, impotente a compiere atti di violenza, ansante sotto l'enorme peso di pensieri rabbiosi. La rivolta non costringeva lui, l'ebreo, a uccidere bensì a gridare alle orecchie dei signori la disperazione di tutti gli schiavi, a distruggere la tranquillità contenta di se stessa, l'immagine del mondo che inganna la certezza, ad ammazzare le menzogne, a persuadere con motivazioni inevitabili. Vincere spiritualmente. Ed egli prende dolore, rivolta, angoscia, fede e li condensa in un discorso violento, toglie il velo della colpa, del peccato dell'uomo e strappa a questa colpa il cuore, strangola la superstizione e vince e vince ${ }^{40}$.

Soffermiamoci su questo estratto: i pensieri riportati acquistano particolare significato se, oltre a rintracciare le premesse teoriche per la successiva adesione al marxismo, si vogliono anche scrutare le affinità col pensiero schopenhaueriano: "l'immagine del mondo che inganna la certezza", "ammazzare le menzogne", "toglie il velo della colpa, del peccato dell'uomo e strappa a questa colpa il cuore, strangola la superstizione" sono immagini in virtù delle quali il riferimento a Schopenhauer appare inevitabile.

In questo senso si deve, dunque, parlare del pessimismo schopenhaueriano come orizzonte all'interno del quale si struttura il discorso marxista in Horkheimer poiché l'idea di un mondo in cui è presente come nocciolo interno l'idea di colpa che è possibile espiare solo squarciando il velo della menzogna e della superstizione - dunque con uno slancio in senso affermativo - è la prima premessa del suo incontro con il marxismo.

Scriverà Horkheimer, molto più in là, in La nostalgia del totalmente Altro:

La dottrina più grandiosa in entrambe le religioni, quella ebraica e quella cristiana, è - e qui mi richiamo ad una parola di Schopenhauer - la dottrina del peccato originale. Essa ha determinato sin qui la storia e ancor oggi la determina per coloro che pensano. Tale dottrina è possibile solo nel presupposto che l'uomo sia stato creato da Dio dotato di libera volontà. La prima cosa che l'uomo fece fu di commettere questo grande peccato nel paradiso, ed è su questa base che tutta la storia dell'umanità necessita di una spiegazione teologica ${ }^{41}$.

40 HORKHEIMER, M. Aus der Pubertät, op. cit., p. 257, tradotto in L. GENINAZZI, Horkheimer\& Co. Gli intellettuali disorganici, op. cit., p. 60.

${ }^{41}$ HORKHEIMER, M. La nostalgia del totalmente Altro, op. cit., p. 78.

Influenze schopenhaueriane nella Sehnsucht del giovane Horkheimer 
Per secoli i gruppi di uomini oppressi hanno dato per evidente e inevitabile che il mondo fosse così strutturato, che irrimediabilmente poggiasse sul privilegio, che non a tutti gli uomini fosse concesso di godere in egual misura di diritti e dignità. Così l'umanità si è assuefatta a quest'ordine sociale dominante, è rimasta per lungo tempo chiusa in questo torpore, senza contemplare la possibilità di un ordine alternativo. Liberare gli uomini dalla menzogna, demistificare la realtà, vincere spiritualmente significò, per Horkheimer, proprio questo: aprire uno squarcio nella rassegnazione e concedere all'umanità l'idea di un'alternativa differente, perseguire l'ideale con coraggio, sollevando i sentimenti degli oppressi verso un fine unico di uguaglianza e parità ed agire conformemente a questo fine. Questo rifiuto del determinismo storico viene ben espresso da Fromm, qualche anno più tardi, in Studi sull'autorità e la famiglia, in particolare nel saggio Masochismo e autorità ${ }^{42}$ :

Il carattere masochistico vive il suo rapporto con il mondo nella prospettiva del destino ineluttabile. Non soltanto ama i fatti che limitano la vita umana e riducono l'umana libertà, ma ama anche sottomettersi a un fato cieco e onnipotente. Quel che gli appare immutabile dipende interamente dalla sua posizione sociale. [..] Crisi e prosperità non sono per lui fenomeni sociali che potrebbero essere mutati dall'intervento umano, ma l'espressione di un governo superiore a cui ci si deve arrendere e piegare. [..] $\grave{E}$ fatale che ci debbano essere guerre; altrettanto ineluttabile che una parte degli uomini debba essere dominata da un'altra o che la quantità di sofferenza nel mondo non possa mai essere considerevolmente minore di quanto non sia fino a oggi. [...] La vita è determinata da potenze che si trovano al di fuori dell'individuo, della sua volontà e dei suoi interessi. Ad esse ci si deve piegare e godere di questa sottomissione è la somma felicità possibile.

Il rifiuto del determinismo fa emergere, così, una personalità volta all'azione, al reale cambiamento delle condizioni sociali che sono all'origine di quell'età complessa, il primo dopoguerra, in cui Horkheimer vive, ventenne. La critica, dunque, cessa di essere

\footnotetext{
42 Il carattere masochistico-autoritario in HORKHEIMER, M. Studi sull'autorità e la famiglia, pp. 104-128. Il carattere autoritario-masochistico di FROMM E., appare per la prima volta con il titolo originale Der autoritär-masochistische Charakter nel volume Studien über Autorität und Familie, edito a Parigi nel 1936 durante il primo anno di permanenza dell'Istituto negli Stati Uniti - poi in Italia nel 1974 con il titolo Studi sull'autorità e la famiglia. Il volume raccoglie i risultati e le considerazioni teoriche derivanti dall'unica ricerca collettiva portata a termine nell'arco dei primi dieci anni di attività dell'Istituto per la ricerca sociale, in particolare dagli studi condotti a Parigi nell'ambito dell'inchiesta empirica sull'autorità, di cui proprio Fromm era coordinatore.

Il carattere autoritario-masochistico è contenuto nella sezione prima, I Saggi Teorici, in corrispondenza della parte sociopsicologica e compare appena dopo Introduzione. Molteplicità dei fenomeni d'autorità; Autorità e Super-Io. Ruolo della famiglia nel loro sviluppo; Autorità e rimozione. 
immobile - «Un pensiero che venga esercitato come fine a se stesso [...]» scrive Horkheimer $^{43}$ - e si unisce alla maturata consapevolezza che a rendere il mondo deprecabile è un assetto sociale fondato sul privilegio e l'ingiustizia; esso è frutto della storia, di una consuetudine umana che può, anzi ora deve, essere modificata. ${ }^{44} \mathrm{La}$ dialettica interna della borghesia, che già in sé contiene i germi della sua contraddizione, si realizza a scapito di un oggetto, il proletariato, che ora deve divenire anche soggetto, forza storica consapevole. Scrive in Crepuscolo: «Solo nella misura in cui gli uomini, esseri radicalmente finiti, riescono ad imporre forme concrete di ragione al mondo storico, questo non rimane più in balìa della natura cieca» ${ }^{45}$.

È una critica che, come abbiamo visto, nasce in seno alla Sehnsucht e passa attraverso la scoperta del dolore del mondo che si oggettiva nelle condizioni brute del proletariato, approdando, così, alla volontà attiva di modificare l'ordine esistente (fondando l'auto-movimento sulla critica immanente) ${ }^{46}$ e giungendo, alla fine, all'adesione al movimento socialista, all'indomani del primo conflitto mondiale. Ciò che appare, però, più significativo in proposito è il rifiuto di un processo storico come sviluppo necessario. Scrive Schmidt:

Non sempre fu visto chiaramente come il pensiero di Horkheimer si muova, fin dalle origini, a partire da una duplice esigenza polemica. Si tratta di opporsi per un verso contro ogni donazione di senso da parte di metafisiche dogmaticamente proclamate; per l'altro contro ogni positivismo che faccia piazza pulita dei concetti, negando astrattamente ogni senso che oltrepassi l'hic et nunc ${ }^{47}$.

Approfondiamo, dunque, il rapporto che vige tra la giovane coscienza infelice che abbiamo visto tra le righe di Aus der Pubertät e il costituirsi, tra la fine della guerra mondiale e gli anni della libera docenza, di questa volontà rivoluzionaria: abbiamo in

\footnotetext{
${ }^{43}$ HORKHEIMER, M. Crepuscolo, Appunti presi in Germania. 1926-1931, op. cit. p. 54.

${ }^{44}$ Cfr. Idem, p. 56.

45 Idem, p. 61. Anche questo piccolo passo è molto interessante poiché presenta la natura come entità maligna in sé, ossia senza l'intervento umano. Questo deve prendere la forma di una ragione alta, libera e incontaminata, che funge da guida per il corso degli eventi, a favore dell'umanità. Con questo Horkheimer non intende certo sposare una tesi di carattere puramente finalistico, quanto piuttosto invitare ad una reale presa di coscienza: il mondo può divenire ospitale per l'uomo, a patto che egli si liberi dal determinismo borghese.

${ }^{46}$ Cfr. MARX, K. Introduzione a Per la critica della filosofia del diritto di Hegelı in La questione ebraica, pp. 94-95. Marx descrive la miseria delle condizioni tedesche e definisce i compiti di una critica immanente, che prende le mosse dall'indignazione e dallo sdegno: «Il suo pathos essenziale è l'indignazione, il suo compito essenziale è la denuncia».

47 HORKHEIMER, M. Taccuini 1950-1969, op. cit., p. 44.

Influenze schopenhaueriane nella Sehnsucht del giovane Horkheimer
} 
precedenza parlato dello sfondo pessimistico-schopenhaueriano che funge da fuoco prospettico per il marxismo horkheimeriano.

Ma si può parlare, come per Schopenhauer, di pessimismo metafisico? In altre parole, qual è il rapporto di Horkheimer con la metafisica? Per rispondere, prendiamo le mosse da un passo contenuto in Crepuscolo:

Un pensiero che venga esercitato come fine a se stesso ha perso il suo significato di essere mezzo per il miglioramento delle condizioni umane. Inoltre [...] la ricerca della verità entrerebbe subito in conflitto con se stessa [...] al posto della realtà si vedrebbe necessariamente costretta a sostituire un fantasma, la verità assoluta, ossia ultraterrena ${ }^{48}$.

In altre parole, per Horkheimer, l'indagine sulla verità che non tiene conto delle condizioni materiali e della condizione di intrinseca fragilità connaturata all'uomo, si conclude inevitabilmente con un ripiegamento su se stessa poiché si troverebbe obbligata a imboccare strade metafisiche. Tutto questo comporta un rischio che Horkheimer ha sempre tenuto a scongiurare: la giustificazione del dolore del singolo in vista di un fine più alto. Scriverà molto più tardi:

Ogni essere finito - e l'umanità è finita - che si pavoneggia come il valore ultimo, supremo ed unico, diventa un idolo, che ha sete di sacrifici di sangue ed inoltre ha il potere demoniaco di assumere un'altra identità e, così, un altro senso ${ }^{49}$.

E ancora

Siamo noi che, mossi ora da paura e sfiducia, ora da speranza, cadiamo nell'errore di contrapporre un'istanza eterna, assolutamente buona e giusta, agli innumerevoli mali del mondo visibile. Tutte le volte che i nostri enunciati oltrepassano la sfera del condizionato e dello storicamente circoscritto, diventano nello stesso tempo "giustificati e ingiustificati. [...] L'insensatezza del mondo smentisce la metafisica, ossia la sua interpretazione sensata ${ }^{50}$.

Il rifiuto di un fondamento sempre valido che sottende la storia, incontra qui il pensiero critico-materialistico, poiché con esso il dolore e le sofferenze degli uomini cessano di essere imputati alla volontà di un ordine superiore. Il terreno di questo

${ }^{48}$ HORKHEIMER, M. Crepuscolo. Appunti presi in Germania, 1926-31, op. cit. p. 54.

${ }^{49}$ HORKHEIMER, M. La Nostalgia del totalmente Altro, op. cit., p. 7.

50 Idem, p. 119.

Influenze schopenhaueriane nella Sehnsucht del giovane Horkheimer 
incontro coincide, quindi, con «la percezione dello stato di dipendenza dell'uomo e quindi con l'impossibilità di dargli una verità assoluta»51.

La metafisica dello spirito oggettivo fu sostituita dalla fede immediata nell'armonia prestabilita degli interessi particolari. Pareva che la mediazione fra l'esistenza empirica del singolo e la coscienza della sua libertà nel tutto sociale, non abbisognasse di alcuna filosofia, ma che fosse sufficiente, a questo scopo, il progresso rettilineo compiuto dalla scienza, dalla tecnica e dall'industria ${ }^{52}$.

L'individuo venne a trovarsi così in una situazione spinosa: abbandonata ogni dimensione metafisica e con essa «la convinzione filosofica di avere la sua vera realtà nell'idea divina incarnata nel tutto», cominciò a leggere il reale con occhi disillusi e pungenti. Non c'era più nulla da indagare sotto la superficie delle cose, nessuna legge che intimamente ne regolasse il cammino, nessun fine futuro e ultimo che potesse in qualche modo fungere da conforto in un presente avverso all'uomo, «la sofferenza e la morte [...] rischiavano di apparire nella loro nudità priva di senso" e Schopenhauer visse "l'aurora della sua filosofia antistorica, pessimistica e benevola ${ }^{53}$. La realtà per com'era sempre stata, con tutte le sue vergogne e le sue miserie, con tutti i suoi attentati ad un'esistenza umana felice e dignitosa, non nascondeva nulla di buono, di alto, di indiscutibile. Era stata completamente spogliata di qualsiasi dimensione escatologica e finalistica.

L'adesione al marxismo avviene allora su un piano storico e si struttura come sintesi tra lotta per il cambiamento dei rapporti sociali e mestizia sullo stato dell'individuo ${ }^{54}$. Scriverà in proposito qualche anno più tardi:

L'inquietudine per la finitezza degli individui e dell'umanità non ha perduto la sua ragion d'essere; ma esse non vanno più nel senso della metafisica, bensì confluiscono nella lotta sociale pratica per una reale sicurezza contro la misera e la morte. Ma la mestizia che ciononostante rimane, si conserva per quello che è e non si lascia più ingannare da alcun sistema ${ }^{55}$.

\footnotetext{
51 Ibidem. Sullo stesso livello si porrà la critica horkheimeriana al positivismo, che al pari di ogni giudizio teologico, non si pone la fondamentale questione del dubbio, ma anzi formula i suoi enunciati con ingenuo ed testardo ottimismo, cosicché teologia e positivismo finiscono, senza sforzi, con l'accettare e giustificare l'esistente.

52 Ibidem.

53 Ibidem.

${ }^{54}$ Cfr. GENINAZZI, Horkheimer \& Co. Gli intellettuali disorganici, op. cit, p. 68.

55 Come giustamente è stato già osservato in proposito da Geninazzi, nell'opera citata M. Horkheimer \& Co. Gli intellettuali disorganici, in questo particolare aspetto del materialismo può essere anche rintracciata la Influenze schopenhaueriane nella Sehnsucht del giovane Horkheimer
} 
Utile per approfondire questo aspetto può risultare il ricorso a quanto scritto da Riconda nel saggio «Horkheimer e Schopenhauer»:

Che Horkheimer abbia per così dire recepito il marxismo sotto un angolo visuale da cui poteva accordarlo con questo suo schopenhauerismo è cosa che non può non qualificare questa stessa recezione: [...] è per questa ragione che egli poté sottrarsi alla suggestione della dialettica nuovamente scoperta da Lucáks e Korsch e con ciò al dogma di un sapere storico totale, al cui servizio tutti i mezzi sono giustificati ${ }^{56}$.

Questa particolare adesione al marxismo, che tanto risente dell'orizzonte filosofico schopenhaueriano, si porrà come base per la teoria critica e per una nuova concezione della cultura materiale, che se nell'originale dottrina marxista era considerata mera sfera sovrastrutturale, con Horkheimer si definisce come terreno in cui viene

prodotto e instillato negli individui il collante che teneva insieme un edificio sociale che meritava di crollare per via della sua intrinseca irrazionalità. La speranza era che quei precari fattori di coesione contenessero anche l'esplosivo che lo avrebbe fatto saltare, che il cemento si tramutasse in dinamite ${ }^{57}$.

\section{Pessimismo e Sehnsucht}

Il peso dell'influenza schopenhaueriana nel complesso della Teoria critica è ben descritto da Schmidt nel passo seguente:

Critica è la teoria di Horkheimer, in quanto vede nel materialismo storico non una Weltanschauung definitiva, ma la denuncia di uno stato - da superarsi - in cui gli uomini, invece di plasmare coscientemente i loro rapporti economici, si lasciano degradare a ciechi organi di esecuzione. Critica - e questo viene ancora più raramente riconosciuto è poi anche in quanto essa con Schopenhauer coraggiosamente afferma che la specie umana, quand'anche fosse più sapientemente organizzata, rimane ancorata ad una radicale finitezza: essa è un che di mistero nel

sua vicinanza col filone illuminista. In altre parole la consapevolezza che questo mondo è il migliore dei mondi possibili viene con Horkheimer assorbita e rielaborata in chiave critico-materialistica.

56 Romanticismo, Esistenzialismo e Ontologia della libertà, in RICONDA, G. «Horkheimer e Schopenhauer».

57 DONAGGIO E. La scuola di Francoforte, la storia e i testi, Einaudi, Torino. 2005, op. cit., p. 23.

Influenze schopenhaueriane nella Sehnsucht del giovane Horkheimer 
$\operatorname{cosmo}^{58}$.

Elementi marxiani ed elementi schopenhaueriani, dunque - i primi in riferimento al malum physicum, i secondi al malum metaphysicum ${ }^{59}$ - si diffondono su tutti i livelli della teoria critica, dal momento che la società giusta continua a rimanere sempre un obiettivo, che però s'intreccia con l'idea di colpa, ${ }^{60}$ e non solo con l'idea di un processo storico scientificamente indagabile.

Il rifiuto di categorie eterne e sfere superiori che legittimino e giustifichino il reale, davanti all'evidenza di una storia ostile all'uomo, fa sì che il pensiero di Horkheimer, e con lui dell'Istituto per la ricerca sociale, si tramuti in un preciso atteggiamento antidogmatico, che si pone a sua volta come premessa peculiare del suo neomarxismo.

Nel materialismo marxiano non esiste, infatti, una sfera superiore, che sovrasta la realtà concreta, e neppure un fondamento assoluto che, come principio unitario, sostiene questa realtà. Il fondamento del materialismo sono i fatti, i quali a loro volta, per essere compresi, abbisognano della filosofia61.

La critica marxistica che Horkheimer propone contro i fondamenti razionali unificanti - che in qualche modo rischiano di essere conciliativi nei confronti della storia - si dirige contro le metafisiche ontologiche che sorreggono un piano superiore alla realtà empirica e contro la filosofia della storia trasfigurante e giustificatrice del reale.

Dunque, pur interiorizzando dolorosamente la consapevolezza della finitudine del cosmo e dell'uomo - come abbiamo visto fin dai tormenti dell'adolescenza condivisi con l'amico Pollock - Horkheimer sceglie di porre questa stessa consapevolezza come

${ }^{58}$ RICONDA, G. «Horkheimer e Schopenhauer», in Romanticismo, Esistenzialismo e Ontologia della libertà, op. cit.

59 SCHMIDT, A. Tugend und Weltlauf. Vorträge und Aufsätze über die Philosophie Schopenhauers (19602003), pp. 12-13 sgg. Cfr. Centro interdipartimentale di ricerca su Arthur Schopenhauer e la sua scuola dell'Università del Salento (a cura di), La scuola di Schopenhauer, Testi e contesti, op. cit. p. 177.

60 Interessante in proposito è ciò che NIETZSCHE, F. in Crepuscolo degli idoli, scrive sullidea schopenhaueriana di colpa: «Spiegazione dei sentimenti comuni sgradevoli. Essi sono determinati da esseri che ci sono ostili [...] Sono determinati come punizioni, come una resa dei conti per qualcosa che non avremmo dovuto fare, non avremmo essere (generalizzato in forma impudente da Schopenhauer in una proposizione, nella quale la morale appare ciò che essa è, la vera avvelenatrice e diffamatrice della vita: «ogni grande dolore, sia fisico, che spirituale, esprime quello che meritiamo; infatti non potrebbe colpirci se non lo meritassimo)».

61 PONSETTO, A. Max Horkheimer, dalla distruzione del mito al mito della distruzione, op.cit. p. 7. 
fondamento della volontà di modificare la realtà esistente. In altre parole egli non rinuncia all'ideale di «una condizione in cui regni un effettivo "dominio degli uomini sulla propria vita oltre che sulla natura" e risulti finalmente sconfitta la "tenebrosa barbarie" che ancora persiste» ${ }^{62}$.

Ma Schopenhauer, per Horkheimer, non è solo un filosofo critico in quanto "lungimirante pessimista"63: egli è colui il quale per primo ha teorizzato il principio della solidarietà tra gli uomini, centrale nella tradizione del marxismo occidentale, senza farlo poggiare su nessuna metafisica consolatoria, né dell'al di là, né dell'al di qua, ma basandolo sulla finitezza dell'uomo e sul suo abbandono su una terra dove non regna più alcuna divinità provvida e benevola64.

Per Horkheimer la giustificazione del processo storico deve, dunque, necessariamente lasciare posto a un pensiero finalmente ancorato a una dimensione sociale:

Vi sono gruppi sociali per i quali la sola chiarezza teoretica sarebbe di impedimento all'adattamento alla situazione data e motivo di conflitto intellettuale [...] Il pensiero concettuale da solo [...] non può distruggere neppure la superstizione più oscura, se questa ha una reale funzione nella dinamica di una struttura sociale appena stabile65.

La teoria, dunque, deve essere incastonata nell'ordine sociale che riflette e che mira ad interpretare, ma soprattutto a correggere tramite un metodo dialettico: «Il metodo dialettico è l'insieme di tutti i mezzi intellettuali, così da formare dagli astratti momenti della ragione parzializzante l'immagine dell'oggetto vivente» 66 .

A questo scopo occorre riaffermare il potere del rifiuto al ricorso a sfere superiori e atemporali, che non tengono conto delle derive negative che la storia, la società umana, l'oggetto vivente di cui parla Horkheimer, hanno assunto nel corso dei secoli.

La realtà non è né una né eterna, gli uomini soffrono e muoiono solo per se stessi e in circostanze diverse. L'affermazione che la realtà nella sua sostanza è indivisibile contraddice il fatto che caratterizza la storia

62 DONAGGIO, E. La scuola di Francoforte, la storia e i testi, op. cit., p. 247.

63 Originariamente tratto da «L'attualità di Schopenhauer», op. cit., pp. 526-545.

${ }^{64}$ Centro interdipartimentale di ricerca su Arthur Schopenhauer e la sua scuola dell'Università del Salento, La scuola di Schopenhauer, Testi e contesti, op. cit. p. 185.

65 HORKHEIMER, M. «A proposito della controversia sul razionalismo nella filosofia attuale», in Teoria critica, op. cit., pp. 119-172.

66 Idem, p. 152.

Influenze schopenhaueriane nella Sehnsucht del giovane Horkheimer 
almeno nella sua forma passata e presente, che l'umanità è scissa in felici ed infelici, dominanti e dominati, sani e malati. I concetti con cui cogliamo questa separazione, le sue cause e i suoi concatenamenti, si sono certo formati con la collaborazione dell'intelligenza ordinatrice e spazializzante; sono soggetti a condizioni storiche, e cioè la loro struttura si fonda sia nella situazione fisica e psichica dei soggetti conoscenti che negli oggetti transitori. [...] ${ }^{67}$.

In questa critica alla filosofia della storia è rintracciabile l'impronta della metafisica pessimistica schopenhaueriana — che non si lascia ridurre a ideologia - e la valorizzazione dell'intransigente nominalismo sociale di Schopenhauer che si rifiuta di considerare gli universali e i concetti generali come realtà anteriori e indipendenti 68 .

Scrive Riconda in proposito, in un saggio dal titolo «Horkheimer e Schopenhauer»:

Egli non cede nulla al mito di Rousseau, rifiuta cioè il mito della nazione, del collettivo che violentemente opprime gli individui, per attenersi invece a [...] uomini e animali presi nella loro singolarità con la loro volontà di felicità e miseria. Valutando ogni realtà da questo punto di vista, egli non paga alcun tributo ad essa interpretandola sullo sfondo d'oro dell'eternità69.

In questo senso possiamo dire che la teoria critica pone come sua premessa fondante l'orizzonte pessimistico. Significativa in proposito è una considerazione contenuta in La scuola di Schopenhauer. Testi e contesti:

Teoria critica significa per Horkheimer "teoria critica della società esistente": essa si basa su di una forma non irrigidita e non dogmatica di materialismo storico, che interpreta Marx alla luce di Hegel, scorgendo nella dialettica il principio dinamico dell'accadere storico, e non disdegna di applicare alla ricerca i risultati della psicanalisi, per cercare di spiegare le forme ideologiche di introiezione del dominio ${ }^{70}$.

Nella teoria critica, dunque, sono ravvisabili:

- $\quad$ il motivo schopenhaueriano della finitezza dell'esistenza umana, ereditato dalla metafisica pessimistica. Questo senso di caducità investe sia l'esistenza del

\footnotetext{
${ }^{67}$ HORKHEIMER, M. «Sulla metafisica bergsoniana del tempo», in Teoria critica, op. cit., vol I, pp. 173-196. ${ }^{68}$ SCHOPENHAUER, A. WWV I / MVR I, p. 541.

${ }^{69}$ RICONDA, G. «Horkheimer e Schopenhauer», in Romanticismo, Esistenzialismo e ontologia della libertà, op. cit. p. 306.

${ }^{70}$ FAZIO, D. M. I contesti in La scuola di Schopenhauer. Testi e contesti, op. cit., p.177. 
singolo uomo - poiché essa inevitabilmente termina con la morte - sia l'umanità in senso collettivo poiché la storia si presenta come un susseguirsi di conflitti violenti e oppressioni, che rispecchiano a loro volta il carattere egoistico ed interessato delle singole relazioni tra gli uomini: «Nell'insistenza con cui Schopenhauer fa valere nella storiografia "ciò che è assolutamente singolare ed individuale" è anche implicita una protesta materialistica contro le metafisiche conformistiche che neutralizzano la sofferenza degli individui, o addirittura la trasfigurano, inserendola in una totalità, che si pretende sensata e significativa, del decorso storico» ${ }^{71}$.

- La potenza demistificatrice della filosofia schopenhaueriana, che lotta, esattamente al pari della teoria critica, contro un «pensiero rassegnato che dipinge il mondo con le tinte del mito» 72 .

- Lo sfondo materialistico che supporta sia la filosofia della storia - che va indagata dialetticamente sul piano della cultura materiale - la quale diventa campo d'indagine e non più, come nel marxismo originale, mera sovrastruttura sia il programma scientifico della teoria critica, che prevede stretta collaborazione tra teoria, prassi e critica all'esistente. La teoria critica è, in questo senso, una filosofia che rinuncia a proporre una consolazione oltremondana e si rivolge al mondo sensibile, al mondo dell'esistente; essa è indiscutibilmente rivolta alla sua consapevole trasformazione.

- Il sottofondo pessimistico, in Horkheimer indissolubilmente legato al materialismo, è di altra natura rispetto al pessimismo rassegnato che non prospetta possibilità di cambiamento. Anzi il materialismo, in quest'ottica, deve partire da un assunto fondamentale, che è quello dell'ineludibile sofferenza del singolo e dello sfruttamento dei gruppi degli oppressi in ogni epoca storica. «Horkheimer è cosciente che vi è pure un pessimismo che assume la forma del fatalismo e della tendenza al declino, ma questo è rifiutato ${ }^{73}$.

\footnotetext{
${ }^{71}$ HORKHEIMER, M. Taccuini 1950-1969, in Prefazione di A. Schmidt, op. cit., 24.

72 DONAGGIO, E. La scuola di Francoforte, La storia e i testi, op.cit., p. 25.

73 GENINAZZI, L. Horkheimer \& Co. Gli intellettuali disorganici, op. cit. p. 72: «Horkheimer ha presente la dialettica insita nell'atteggiamento pessimista, nella sfiducia verso le masse di Schopenhauer: "La sfiducia nel movimento popolare racchiude anche nel XIX secolo non solo un elemento reazionario, ma, al pari del pessimismo sociale di cui fa parte, anche un momento di consapevolezza. Nell'amarezza del pessimismo si cela il sospetto che nella società vigente, nonostante la assicurazione dei suoi apologeti, la felicità generale abbia ben poche possibilità di realizzazione"». 
La teoria critica, quindi, conduce, attraverso la composizione in chiave organica e compiuta dei punti precedentemente esposti, alla presa di coscienza del profondo nesso tra teoria e prassi, al rifiuto di ogni ideologia o filosofia dogmatica della storia che trasfigura il reale e che, dunque, si pone come strumento di indagine acritico, recuperando, in tal senso, la dimensione storico-materiale della ragione e assegnando al processo storico di emancipazione, una guida teorica e intellettuale, la ricerca sociale, appunto.

Nel 1970, a tre anni dalla sua morte, in La nostalgia del totalmente Altro, Horkheimer ritorna, come per un cerchio che si chiude, a parlare di Sehnsucht.

Questa aspirazione all'ideale di giustizia, che permea costantemente la sua filosofia, nasce, come abbiamo visto, dall'esperienza del dolore, dal senso di abbandono nel cosmo, dalla penosa consapevolezza della finitudine del mondo. Che questa esperienza del dolore, tipica del romanticismo tedesco, poi riconfermata e perfezionata in Schopenhauer, abbia influito profondamente su tutto il suo itinerario intellettuale è indubbio: la percezione di un distacco insanabile dal mondo borghese, la volontà di mutare le condizioni materiali che realizzano l'ingiustizia sociale e il dominio dell'uomo sull'uomo, sono le conseguenze di una particolare sensibilità intellettuale nei confronti della miseria, dell'ingiustizia e del dolore. È questa la più compiuta forma di eredità schopenhaueriana nella filosofia in Horkheimer.

Alfred Schmidt si spingerà oltre quando dirà che, nell'ultima fase del pensiero horkheimeriano, il pessimismo schopenhaueriano verrà addirittura oltrepassato:

Schopenhauer, nell'essenziale, era ancora un ottimista. Il dolore non è stato negato neanche dagli esponenti ufficiali dell'ottimismo, compresi Leibniz e Hegel, che ci hanno semplicemente aggiunto la favola rassicurante della metafisica dogmatica. [...] L'ottimismo metafisico di Schopenhauer diventa evidente soprattutto quando egli accetta il mito della metempsicosi, dove si afferma non solo che le singole anime hanno un destino trascendente diverso, ma anche che per alcune di esse sussiste la possibilità reale di un riscatto finale. La separazione dall'uno verrebbe, così, meno. Ci vuole fede, e molta, per credere che la categoria dell'unità sia meno illusoria, meno fenomenica di quella della molteplicità, che la proiezione del monarca sia più reale della proiezione dell'aristocrazia e del liberalismo. [...] Comunque un essere umano si immagini la fine del dolore, la morte e la risurrezione, a qualsiasi cosa attribuisca una realtà assoluta, all'amore celeste o all'amore terreno, si tratta sempre di un attimo di cattiva infinità. Il buon infinito è soltanto un precario conforto filosofico. Così Schopenhauer ha ragione contro se 
stesso. [...] Che l'esperienza della quintessenza del mondo diventi un quietivo, è un processo psicologico, non metafisico. Il dolore è eterno ${ }^{74}$.

\section{Riferimenti bibliografici}

ADORNO, T. W. In Empirische Sozialforschung, Wissenschaftliche Schriftenreihe des Instituts zur Förderung öffentlicher fini. Angelegenheiten Zur gegenwärtigen Stellung der empirischen Sozilaforschung in Deutschland; e V., Bd. XIV, Frankfurt a. M., 1952.

BEDESCHI, G. Introduzione alla Scuola di Francoforte. Roma-Bari: Editori Laterza, 2005.

BEMJAMIN, W. Berliner Kindheit um neunzehnhundert. Trad. it. Torino: Einaudi, 1973.

BETTINI, F. Teorie letterarie nella Scuola di Francoforte. Roma: Savelli, 1976.

CENTRO INTERDIPARTIMENTALE DI RICERCA SU ARTHUR SCHOPENHAUER E LA SUA SCUOLA dell'Università del Salento (a cura di). La scuola di Schopenhauer: testi e contesti. Lecce: Pensa Multimedia, 2009.

CIRACÌ, F. Il mondo come volontà, idee e rappresentazione. Per una possibile lettura in senso illuministico della dottrina delle idee. Revista Voluntas: estudos sobre Schopenhauer, Rio de Janeiro, I sem. 2010, vol. 1, n. 1, pp. 71-115.

DONAGGIO, E. La scuola di Francoforte, la storia e i testi. Torino: Einaudi, 2005.

FROMM, E. La crisi della psicanalisi. Milano: Mondadori, 1971.

GALEAZZI, U. La scuola di Francoforte: teoria e critica in nome dell'uomo. Roma: Città nuova, 1975.

GENINAZZI, L. Horkheimer \& Co. Gli intellettuali disorganici. Milano: Jaca Book, 1977.

HESSE, H. Demian. Milano: Oscar Mondadori Moderni, 2007.

HORKHEIMER, M.; ADORNO, T. W. Lezioni di sociologia, Istituto per la ricerca sociale di Francoforte. Torino: Einaudi, 1966.

HORKHEIMER, M. Aus der Pubertät. Novellen und Tagebuchblätter. München, 1974.

HORKHEIMER, M. Crepuscolo. Appunti presi in Germania, 1926-1930. Trad. it. di G. Backhaus. Torino, 1977.

HORKHEIMER, M. La nostalgia del totalmente Altro. Trad. it. Querianiana. Brescia, 2001.

HORKHEIMER, M. Studi di filosofia della società. A cura di Werner Brede. Torino: Einaudi, 1981.

HORKHEIMER, M. Taccuini 1950-1969. Genova: Marietti, 1988.

HORKHEIMER, M. Teoria critica. 2 voll. Trad.it. di G. Backhaus. Torino, 1974.

MANN, T. Tönio Kroger, La morte a Venezia, Tristano. Milano: Feltrinelli, 1965.

NIETZSCHE, F. Schopenhauer educatore. Trad. it di V. Arangio-Ruiz. Milano: All'insegna del pesce d'oro, 1973.

PONSETTO, A. Max Horkheimer, dalla distruzione del mito al mito della distruzione. Bologna: Il Mulino, 1981.

RICONDA, G. In Romanticismo, Esistenzialismo e Ontologia della libertà, Horkheimer $e$ Schopenhauer. Milano: U. Mursia, 1979.

ROSEN, Z. Max Horkheimer. München: C. H. Beck, 1995.

RUSCONI, G. E. La scuola di Francoforte, origini e significato attuale. Bari: De Donato, 1972.

SCHMIDT, A. Zur Idee der Kritischen Theorie, Elemente der Philosophie Max Horkheimer. München, 1974.

SCHOPENHAUER, A. Il mondo come volontà e rappresentazione. Trad. it. S. Giametta. Milano: BUR Biblioteca Univ. Rizzoli, 2002.

SCHOPENHAUER, A. Parerga e paralipomena. A cura di G. Colli. Milano: Adelphi, 1981.

VINCIERI, P. Discordia e destino in Schopenhauer. Genova: Il Melangolo, 1993.

WIGGERSHAUS, R. La Scuola di Francoforte. Storia. Sviluppo teorico. Significato politico. Torino: Bollati Boringhieri, 1992.

${ }^{74}$ HORKEIMER, M. Taccuini 1950-1969, op. cit., p. 162.

Influenze schopenhaueriane nella Sehnsucht del giovane Horkheimer 
Recebido: 05/05/17 Received: 05/05/17

Aprovado: 02/07/17 Approved: 07/02/17 